\title{
Expanding hot flow in the black hole binary SWIFT J1753.5-0127: evidence from optical timing
}

\author{
Alexandra Veledina ${ }^{1,2 \star}$, Poshak Gandhi ${ }^{3}$, Robert Hynes ${ }^{4}$, Jari J. E. Kajava ${ }^{2,5}$, \\ Sergey S. Tsygankov ${ }^{2,6}$, Michail G. Revnivtsev ${ }^{6}$, Martin Durant ${ }^{7}$, and Juri Poutanen ${ }^{1,2}$ \\ ${ }^{1}$ Nordita, KTH Royal Institute of Technology and Stockholm University, Roslagstullsbacken 23, SE-10691 Stockholm, Sweden \\ ${ }^{2}$ Tuorla Observatory, University of Turku, Väisäläntie 20, FI-21500 Piikkiö, Finland \\ ${ }^{3}$ Department of Physics \&S Astronomy, University of Southampton, Highfield, Southampton SO17 1BJ, UK \\ ${ }^{4}$ Department of Physics and Astronomy, Louisiana State University, Baton Rouge, LA 70803-4001, USA \\ ${ }^{5}$ European Space Astronomy Centre (ESA/ESAC), Science Operations Department, 28691 Villanueva de la Cañada, Madrid, Spain \\ ${ }^{6}$ Space Research Institute of the Russian Academy of Sciences, Profsoyuznaya Str. 84/32, Moscow 117997, Russia \\ ${ }^{7}$ Department of Medical Biophysics, Sunnybrook Hospital M6 623, 2075 Bayview Avenue, Toronto M4N 3M5, Canada
}

15 November 2016

\begin{abstract}
We describe the evolution of optical and X-ray temporal characteristics during the outburst decline of the black hole X-ray binary SWIFT J1753.5-0127. The optical/Xray cross-correlation function demonstrates a single positive correlation at the outburst peak, then it has multiple dips and peaks during the decline stage, which are then replaced by the precognition dip plus peak structure in the outburst tail. Power spectral densities and phase lags show a complex evolution, revealing the presence of intrinsically connected optical and X-ray quasi-periodic oscillations. For the first time, we quantitatively explain the evolution of these timing properties during the entire outburst within one model, the essence of which is the expansion of the hot accretion flow towards the tail of the outburst. The pivoting of the spectrum produced by synchrotron Comptonization in the hot flow is responsible for the appearance of the anti-correlation with the X-rays and for the optical quasi-periodic oscillations. Our model reproduces well the cross-correlation and phase lag spectrum during the decline stage, which could not be understood with any model proposed before.
\end{abstract}

Key words: accretion, accretion discs - black hole physics - stars: individual: SWIFT J1753.5-0127 - X-rays: binaries.

\section{INTRODUCTION}

The properties of accreting black holes (BHs) in X-ray binaries have been studied for almost half a century. Most investigations relied on X-ray diagnostics. Spectra and their evolution, variability on the timescales as short as milliseconds and as long as months, and interconnection of different energy bands allowed us to probe the regions in the immediate vicinity of the compact object (Zdziarski \& Gierliński 2004; Remillard \& McClintock 2006; Gilfanov 2010; Belloni \& Stella 2014; Poutanen \& Veledina 2014). We know that the bulk of the X-rays are produced very close to the $\mathrm{BH}$, probably, within $10 R_{\mathrm{S}}$ (where $R_{\mathrm{S}}=2 G M / c^{2}$ is the Schwarzschild radius and $M$ is the black hole mass), in an optically thin and hot medium characterised by a Thomson optical depth of about unity and an electron temperature

^ E-mail: alexandra.veledina@su.se of $\sim 100 \mathrm{keV}$. Some of these primary X-rays are reflected from the surface of the cold thin disc producing characteristic features such as a fluorescent iron line and spectral hardening above $10 \mathrm{keV}$. The location of the hot medium above the cold thin disc or within its truncated radius - was debated for several decades (Done, Gierliński \& Kubota 2007). A related question concerns the source of seed photons for Comptonization which may come either from the cold disc (e.g., Poutanen, Krolik \& Ryde 1997) or from the synchrotron emission internally produced in the hot medium itself (Esin et al. 1997; Poutanen \& Vurm 2009; Malzac \& Belmont 2009). Many observables suggest the cold disc is truncated far away from the black hole in the hard state, at about $100 R_{\mathrm{S}}$ : the characteristic suppression of the variability amplitude of the reflected emission compared to the primary X-rays (Gilfanov, Churazov \& Revnivtsev 2000; Revnivtsev, Gilfanov \& Churazov 2001), the decrease of the iron line equivalent width with increas- 


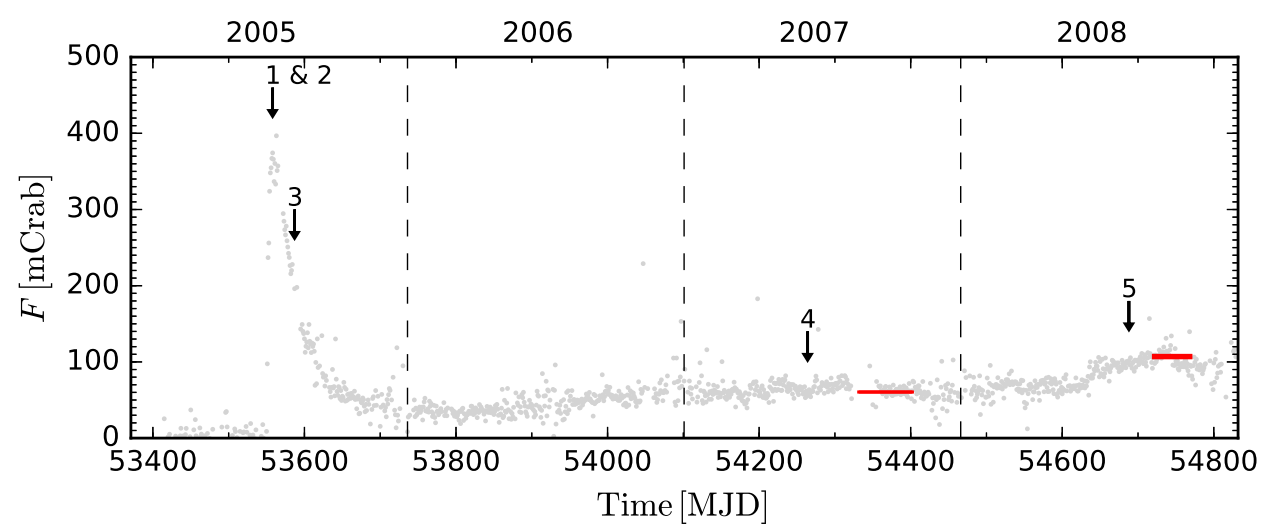

Figure 1. The 15-50 keV Swift/BAT light-curve of the BH binary SWIFT J1753.5-0127. The epochs considered in this work are shown with arrows. Red stripes mark the INTEGRAL/ISGRI data used for spectral fitting (for epochs 4 and 5). High-energy spectra for epochs 1,2 and 3 are obtained from the RXTE/HEXTE data taken at the same time as RXTE/PCA.

ing Fourier frequency, X-ray luminosity and spectral hardness (Revnivtsev, Gilfanov \& Churazov 1999; Plant, Fender, Ponti, Muñoz-Darias \& Coriat 2015; Basak \& Zdziarski 2016), the low temperature of the cold disc (Frontera et al. 2001; Chaty et al. 2003) and reverberation lags (De Marco et al. 2015; De Marco \& Ponti 2016). Recently, yet another piece of evidence was presented. This comes from the X-ray low-frequency quasi-periodic oscillations (QPOs), which were shown to be consistent with the Lense-Thirring precession of the hot medium by the detection of characteristic variations of the iron line blue/red shifts as the approaching or receding side of the cold disc is illuminated (Ingram et al. 2016). The flow precesses as a whole only under the condition of hot accretion (Fragile, Blaes, Anninos \& Salmonson 2007; Ingram, Done \& Fragile 2009), and its characteristic frequency depends on the outer radius of the hot flow, which coincides with the truncation radius.

Because of the large amount of data and detailed studies of them, the X-ray information provided a reference point for studies in other wavelengths. Whenever the variability in the optical, infrared or ultraviolet range (hereafter, just optical) was investigated, it was compared to or contrasted with the behaviour in the X-rays. Three components can potentially contribute to the optical emission: the cold optically thick accretion disc, the hot optically thin X-ray emitting medium and an outflow/jet (see review by Poutanen \& Veledina 2014). To break the spectral fitting degeneracy, timing information is used. Simultaneous optical/X-ray data were obtained which, however, revealed another mystery: some observations showed a positive correlation with optical photons lagging the X-rays, consistent with simple reprocessing (Hynes et al. 1998, 2003, 2009), other demonstrated a very broad and nearly symmetric positive cross-correlation (Casella et al. 2010), while in a number of cases a more complex structure containing a so-called precognition dip (anti-correlation) at negative lags (optical photons leading X-rays) was observed (Motch et al. 1983; Kanbach et al. 2001; Gandhi et al. 2008, 2010; Durant et al. 2008, 2011).

In addition to the broadband variability, QPOs were identified in the optical power spectra (e.g., Motch et al.
1983; Hynes et al. 2003; Durant et al. 2009; Gandhi et al. 2010). They appear to share common frequencies with the X-ray QPOs for months of observations (Hynes et al. 2003), and it was recently shown that they are phase connected (Veledina et al. 2015; Kalamkar et al. 2016).

In this work we analyse several simultaneous optical/Xray data sets obtained for the BH binary SWIFT J17530127. The object underwent an outburst in the middle of 2005 and was active for over 11 years. It is now returning to the quiescent state (Shaw et al. 2016b). The first four years of the outburst light-curve and the analysed epochs are shown in Fig. 1. This unique X-ray dataset revealed many interesting phenomena. The temporal properties of the source, in particular, the correlation of the X-ray and optical light-curves, reveal dramatic changes throughout the outburst (see Fig. 2, Hynes et al. 2009; Durant et al. 2008, 2011). Recently, the spectral evolution throughout the ten years of the outburst was analysed (Kajava et al. 2016), with the aim of placing constraints on the accretion geometry and relevant radiative processes in this object. The dependence of the ratio of the Comptonized to disc luminosity on the spectral index revealed that the data points follow two different tracks above and below a critical flux. At higher fluxes, the spectra are well explained by disc Comptonization, however, at lower fluxes, the inferred (observed) Comptonization luminosity is too high to be originating from upscattered disc photons. These spectra are instead well reproduced by the Comptonization of synchrotron photons selfgenerated within the hot flow, implying a scenario where the cold disc recedes from the $\mathrm{BH}$ as the outburst proceeds. Infrared/optical spectral energy distribution is well described by a power-law with $F_{\nu} \propto \nu^{1}$ (Froning et al. 2014; Rahoui et al. 2015; Tomsick et al. 2015), which is consistent with the synchrotron emission from the stratified hot flow (Veledina et al. 2013a; Poutanen et al. 2014). Interestingly, the object recently demonstrated several short incursions into the soft state at very low luminosities (Yoshikawa et al. 2015; Shaw et al. 2016), challenging the simple one-to-one relation of the disc truncation radius and the X-ray luminosity. The accretion history is likely an important factor in such relations.

In this paper we present the study of the evolution of 
the variability properties in SWIFT J1753.5-0127. We show that the temporal characteristics of the source can be explained by the expanding hot accretion flow, which emits in the optical through X-rays. We investigate the evolution of power spectral densities (PSDs), cross-correlation functions (CCFs) and phase lag spectra and find that the optical emission can arise from simple reprocessing at the outburst peak, that it has an additional synchrotron component in the outburst tail, and that the decline stage can also be reproduced if the X-rays are produced by both synchrotronand disc Comptonization. Our findings support a picture where the cold accretion disc is receding towards the tail of the outburst, and a hot accretion flow is developing within its truncation radius.

\section{DATA}

We reanalyse the optical data obtained on 2005 July 6 and 7 with the Argos CCD photometer on the McDonald Observatory 2.1-m telescope (Nather \& Mukadam 2004), on 2005 Aug 9 with the FORS2 instrument on the VLT/UT2 telescope using HIT fast imaging mode (O'Brien 2008) and on 2007 June 13 and 2008 Aug 10 with ULTRACAM (Dhillon et al. 2007) mounted on the VLT/UT3 telescope. Simultaneous exposures were taken with Rossi X-ray Timing Explorer (RXTE)/PCA in each case. The X-ray data were analysed with the help of HEASOFT. Light-curves were created using the XRONos package. The X-ray PSDs were normalised according to Leahy et al. (1983) and the Poisson noise was subtracted. The optical PSDs have arbitrary normalisation, no noise was subtracted from the data.

The data were published in Hynes et al. (2009) and Durant et al. (2008, 2009, 2011). We briefly describe the data highlighting the features important for our modelling. We further use the following notations: the outburst peak refers to 2005 July 6 and 7 (epochs 1 and 2 in Fig. 1, respectively), the outburst decline (stage) denotes 2005 Aug 9 (epoch 3), and the outburst tail refers to 2007 June 13 and 2008 Aug 10 (epochs 4 and 5, respectively). For the combined characteristics, such as CCF, cross-spectra and phase lags, we use simultaneous segments of data, and for PSDs we use longer light-curves with non-simultaneous segments, whenever possible (a detailed description of the calculation of these characteristics and their physical meaning can be found in Bendat \& Piersol 1986; Nowak, Wilms \& Dove 1999). The optical light-curves during the outburst tail were obtained by dividing, for each time-bin, the target flux by the flux of the comparison star. For this reason, we use an arbitrary normalisation of the optical PSD. In order to eliminate the long-term variations in the optical light-curve, we subtract a linear trend. This procedure, however, does not eliminate the contamination of the atmospheric noise, which is apparent in the optical PSDs.

The light-curves were split into segments; CCF, PSDs and cross-spectra were calculated in each of them, and then averaged over the segments. The cross-spectra were computed as the product of optical and conjugated X-ray Fourier images. These are vectors on the complex plane, so we apply vector averaging procedure. We plot the lengths of these vectors (denoted as CSD in figures and called just crossspectra hereafter) and their angles on the complex plane (the phase lag spectra, $\Delta \phi$ ), which are determined in the interval $(-\pi, \pi]$. The phase lags have $2 \pi$ uncertainty. Positive phase lags correspond to the delays of the optical light-curve with respect to the X-rays. The cross-spectra show how the variability power of the correlated part of the signal is distributed over the Fourier frequencies $f$, while the phase lags show how the variability in one band is delayed with respect to another at each frequency. Sometimes, the time lags $(\Delta t)$ are a more intuitive representation of the delays, which are connected to phase lags through $\Delta t=\Delta \phi / 2 \pi f$. The errors are estimated using bootstrap method with $10^{5}$ trials.

\subsection{Outburst peak}

The simultaneous optical $V$ and X-ray data were taken during the outburst peak. The optical light-curves have $1 \mathrm{~s}$ time resolution. The X-ray light-curves were obtained from Standard 1 mode with $0.125 \mathrm{~s}$ time resolution in the energy range $2-60 \mathrm{keV}$. For each night, the data were split into 25 segments, $128 \mathrm{~s}$ long each. The resulting characteristics are shown in Fig. 2 (in black and red for July 6 and 7, respectively).

The two X-ray PSDs are very similar and can be represented by a zero-centred Lorentzian (Nowak et al. 1999; Belloni et al. 2002). The optical PSDs constitute a bump at frequencies below $\sim 0.1 \mathrm{~Hz}$, and are flat at higher frequencies, which are likely dominated by the noise. The CCF structure does not change within these two nights, however, its amplitude is somewhat reduced on July 7 . The phase lags reveal a slowly increasing trend, again, nearly identical within two nights. The CCF shape suggests there is one component in the optical which is positively correlated and delayed with respect to the X-rays, and the delay time is in the range expected from thermal reprocessing (Hynes et al. 2009), making it a plausible source of optical variability.

\subsection{Outburst decline}

The $V$-band optical light-curve consists of several distinct segments. We choose 11 segments which are simultaneous with the X-rays $(3-20 \mathrm{keV})$, each lasting $64 \mathrm{~s}$. The time resolution is $0.25 \mathrm{~s}$ for the optical light-curve and $0.01 \mathrm{~s}$ for the X-rays. The results are plotted in Fig. 2 (green lines).

The CCF during the decline stage consists of an anticorrelation dip at time lags between $-3 \mathrm{~s}$ and $3 \mathrm{~s}$, followed by two peaks at $\sim 4$ and $8 \mathrm{~s}$. Such CCF was not seen in any other object. We calculate the CCFs for different X-ray energy ranges and find that they are nearly identical. The X-ray PSD demonstrates a QPO at $\sim 0.25 \mathrm{~Hz}$, but no apparent optical QPO can be seen. The QPO period is the same as the separation of positive peaks seen in the CCF, so the second peak is likely arising from an aliasing, as was suggested in Hynes et al. (2009). In addition to that, we note a hump at $\sim 0.6-0.7 \mathrm{~Hz}$ (above the possible QPO harmonic), which was previously modelled as an additional broad QPO (Hynes et al. 2009). The phase lag spectrum is a steep function of Fourier frequency. It crosses the $\pi$ discontinuity point at least once, at $\sim 0.07 \mathrm{~Hz}$, so we subtract $2 \pi$ from phase lags for the sake of clarity. Dramatic change of the CCF shape, now demonstrating an anti-correlation and double-peak structure at positive lags, suggests an alternative mechanism responsible for the optical emission, as 

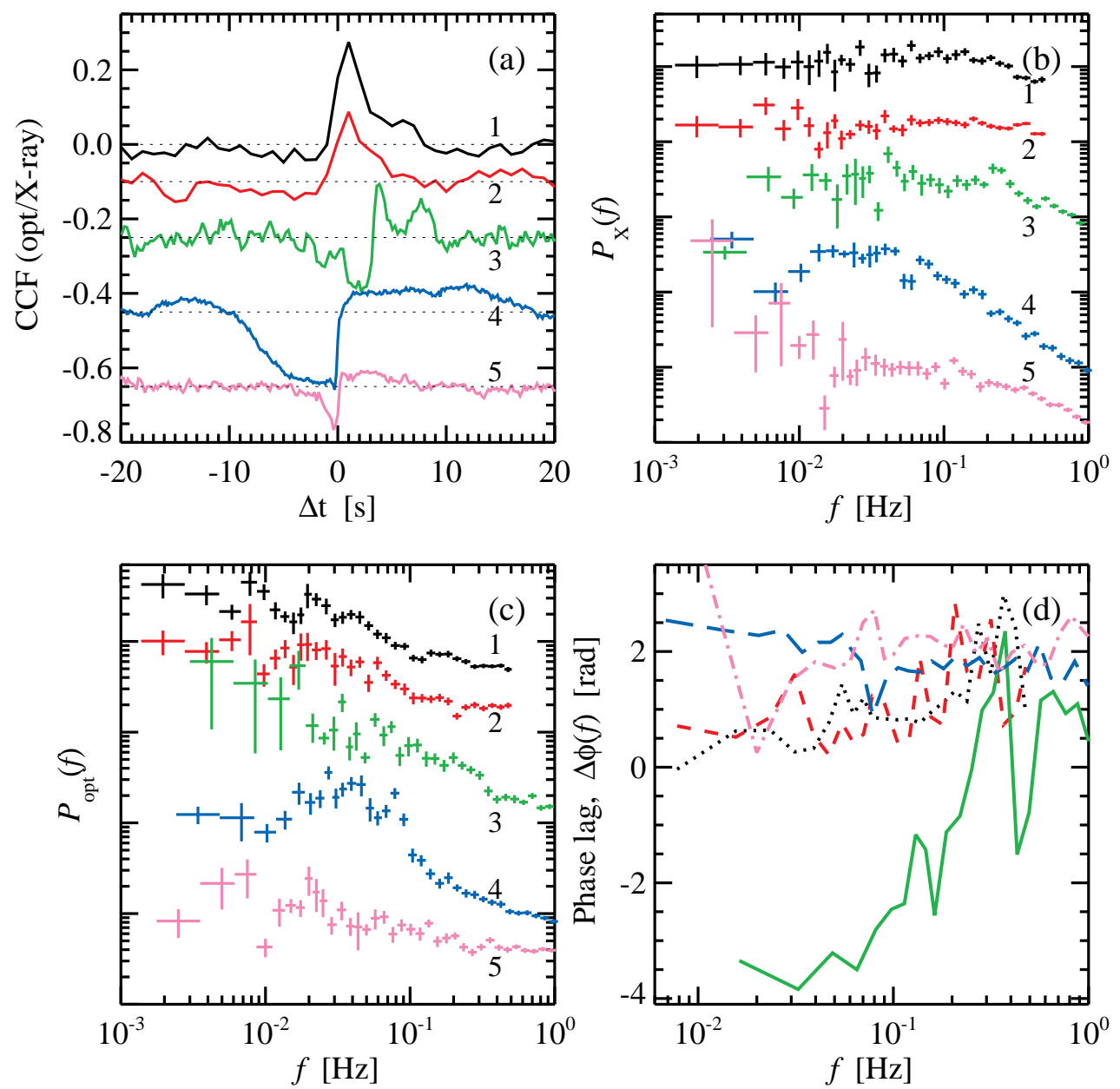

Figure 2. (a) Optical/X-ray CCFs (shifted vertically for clarity), arbitrary normalised (b) X-ray and (c) optical PSDs, (d) optical/X-ray phase lags from different outburst stages top to bottom: 2005 July 6 (epoch 1, black lines, dotted line in panel d), July 7 (epoch 2, red, short-dashed), Aug 9 (epoch 3, green, solid), 2007 June 13 (epoch 4, blue, long-dashed), 2008 Aug 10 (epoch 5, magenta, dot-dashed). Positive time-lags in panel (a) correspond to lag of optical photons and dotted lines indicate zero level for each CCF. We subtract $2 \pi$ from the 2005 Aug 9 phase lag spectrum in panel (d) at frequencies below $\sim 0.07 \mathrm{~Hz}$ for clarity. The evolution of CCF shape is apparent.

simple disc reprocessing is no longer able to reproduce the observed $\mathrm{CCF}$ and phase lags.

\subsection{Outburst tail}

Two simultaneous optical (in filters $g^{\prime}$ and $r^{\prime}$ ) and X-ray $(2-20 \mathrm{keV})$ datasets were obtained during the outburst tail. We show the analysis for $r^{\prime}$ filter, because the $g^{\prime}$ demonstrates nearly identical behaviour. The detailed analysis and modelling of the 2007 dataset is presented by Veledina et al. (2015); we repeat the main results here for the sake of completeness. The simultaneous light-curves were split into 20 segments, each of about $146 \mathrm{~s}$ long. Both light-curves have time resolution $0.143 \mathrm{~s}$. The resulting CCF, PSDs and phase lags are shown in Fig. 2 (blue lines). Now the CCF has one pronounced dip at negative lag, followed by a pronounced peak at positive lags. In addition to that, it demonstrates the oscillating behaviour which we attribute to the QPO at $f=0.08 \mathrm{~Hz}$, apparent in optical and in the X-ray PSD. The phase lags are almost independent of frequency, but show a significant decrease at the QPO frequency.
The 2008 light-curves have a $0.195 \mathrm{~s}$ time resolution. We use 24 segments, each of about $100 \mathrm{~s}$. The results are shown in Fig. 2 (magenta lines). The CCF shows a single dip plus peak structure; no other structures can be seen. Neither optical nor X-ray QPOs are apparent in the PSDs. The amplitudes of the dip and the peak are somewhat lower, which might be due to a larger contribution of atmospheric noise as compared to the previous case. The white highfrequency $(\gtrsim 0.2 \mathrm{~Hz})$ noise is also apparent in the optical PSD. The phase lags increase with frequency, but show a kink at $\sim 0.4 \mathrm{~Hz}$.

Both datasets obtained during the outburst tail are in agreement with a model where optical emission consists of two components: synchrotron emission from the hot accretion flow and the irradiated disc emission (Veledina, Poutanen \& Vurm 2011b). Whether this model can also account for the temporal properties during the decline stage is not immediately clear. We further investigate this possibility below. 


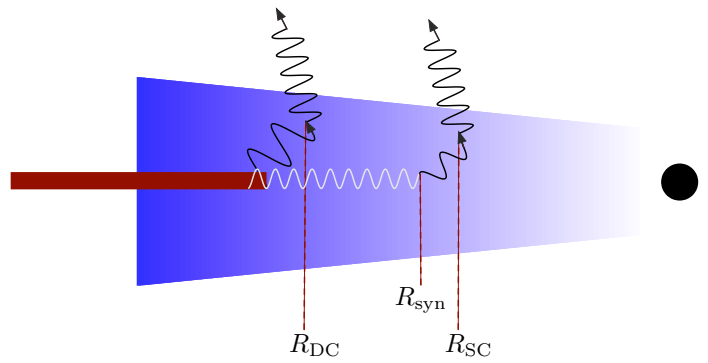

Figure 3. Sketch of the geometry considered. The oscillations in white represent propagating fluctuations in the mass accretion rate, which are eventually transformed to radiation (black lines). $R_{\mathrm{DC}}$ is the radius at which disc photons Comptonize, $R_{\mathrm{syn}}$ is the characteristic radius where the optical synchrotron photons are emitted and $R_{\mathrm{SC}}$ is the radius where synchrotron Comptonization proceeds.

\section{MODEL}

\subsection{General picture}

In this section we describe the model used for fitting of the computed characteristics (see also Veledina et al. 2011b, 2015). It is generally accepted that the X-rays are produced in the hot medium close to the $\mathrm{BH}$, while the optical radiation often consists of the irradiated disc contribution together with synchrotron emission from the hot flow itself (Poutanen \& Veledina 2014). The X-ray broadband variability is driven by the fluctuations at a range of radii. Each radius mainly contributes to one particular frequency, and the accumulated variability from larger radii propagate through the flow towards the compact object, thus the radiation produced at particular radius varies at frequencies up to the characteristic frequency of this radius (Lyubarskii 1997; Kotov, Churazov \& Gilfanov 2001; Churazov, Gilfanov \& Revnivtsev 2001; Arévalo \& Uttley 2006; Ingram \& Done 2011).

The X-ray emission is produced in the region of maximal energy release, within about $10 R_{\mathrm{S}}$. It is commonly accepted to originate from Compton up-scattering, but the source of seed photons is debated. In the soft state, the bulk of soft photons for Comptonization is likely provided by the disc. In the hard state, the fraction of disc photons available for Comptonization is dramatically decreased because of the large truncation radius, so the synchrotron emission self-generated in the hot flow becomes an important, or even dominant, source of seed photons (Poutanen et al. 1997; Esin et al. 1997; Poutanen \& Vurm 2009; Malzac \& Belmont 2009, Veledina, Poutanen \& Vurm 2013a). It is then likely that both sources coexist during the intermediate state (Veledina 2016).

In the propagating fluctuations model, the accretion rate changes first drive the disc Comptonization variability (close to the truncation radius) and then they propagate into the hot flow, exciting the X-ray fluctuations again, this time via synchrotron Comptonization (see Fig. 3). We assume that the variations in accretion rate are directly mapped into the broadband fluctuations of the disc- and synchrotron Comptonization continua. These two mechanisms are independent of each other and so their luminosities are additive. Moreover, the disc Comptonization likely proceeds close to the truncation radius, hence its light-curve lacks the high- frequency variations generated closer to the $\mathrm{BH}$. We simulate this by introducing a low-pass filter function with the Fourier image

$H(f)=\frac{1}{\left(f / f_{\text {filt }}\right)^{2}+1}$,

where $f_{\text {filt }}$ is the characteristic frequency of fluctuation power damping, which is determined by the radius at which the disc photons are effectively up-scattered (we use small letters with argument $t$ to denote variables in time domain and capital letters with argument $f$ for those in the frequency domain hereafter). The disc Comptonization lightcurve is obtained from the convolution of the mass accretion rate light-curve with the filter function. The aperiodic synchrotron Comptonization light-curve is assumed to be proportional to the mass accretion rate light-curve.

The broadband variability is accompanied by the low-frequency QPOs, which can arise from the LenseThirring precession of the entire hot flow (Fragile et al. 2007; Ingram et al. 2009, 2016). We consider the model where the QPOs appear because of the anisotropy of the hot flow emission (Veledina, Poutanen \& Ingram 2013b, Poutanen \& Veledina, in prep.). The instantaneous emitted X-ray flux is determined by the current mass accretion rate, and to obtain the observed flux, we need to account for the angular distribution of radiation by multiplying the emitted power by the emission diagram (and also by the instantaneous solid angle occupied by the hot flow on the observers sky). As the precession proceeds, the inclination angle of the flow changes resulting in the modulation of the observed X-ray flux. Hence, the QPO modulates the accretion rate fluctuations in a multiplicative way.

The X-ray emission is reprocessed in the outer parts of the cool disc giving rise to the optical emission. In addition to the disc, the hot flow synchrotron emission also contributes to optical wavelengths. The optical light-curve is a weighted sum of these two components.

\subsection{Mathematical formulation}

The description we have introduced for the X-ray $x(t)$ and optical $o(t)$ light-curves can be expressed mathematically as

$x(t)=\varepsilon_{\mathrm{m}} \dot{m}\left(t+t_{0}\right) * h(t)+[1+\dot{m}(t)]\left[1+\varepsilon_{\mathrm{x}} q(t)\right]-1$,

$o(t)=s(t)+r_{\mathrm{ds}} d(t)$,

where $\dot{m}(t)$ is the mass accretion rate light-curve, $h(t)$ is the filter function, $*$ sign denotes convolution, $q(t)$ describes the QPO light-curve, $s(t)$ and $d(t)$ are the synchrotron and irradiated disc light-curves, respectively. All the light-curves have zero mean and represent deviations from the average quantity. In the equation for the X-ray light-curve, the first term corresponds to the disc Comptonization and the term in brackets describes the emission coming from synchrotron Comptonization. The parameter $\varepsilon_{\mathrm{m}}$ regulates their relative importance and $t_{0}$ is the delay between the disc and the synchrotron Comptonization processes. The positive parameter $\varepsilon_{\mathrm{x}}$ gives the relative importance of the QPO process and $r_{\mathrm{ds}}$ describes the relative importance of the disc and the synchrotron components. The power spectra of both the synchrotron and disc light-curves are normalised so that their integrals are equal, thus $r_{\mathrm{ds}}$ gives the ratio of the total root 

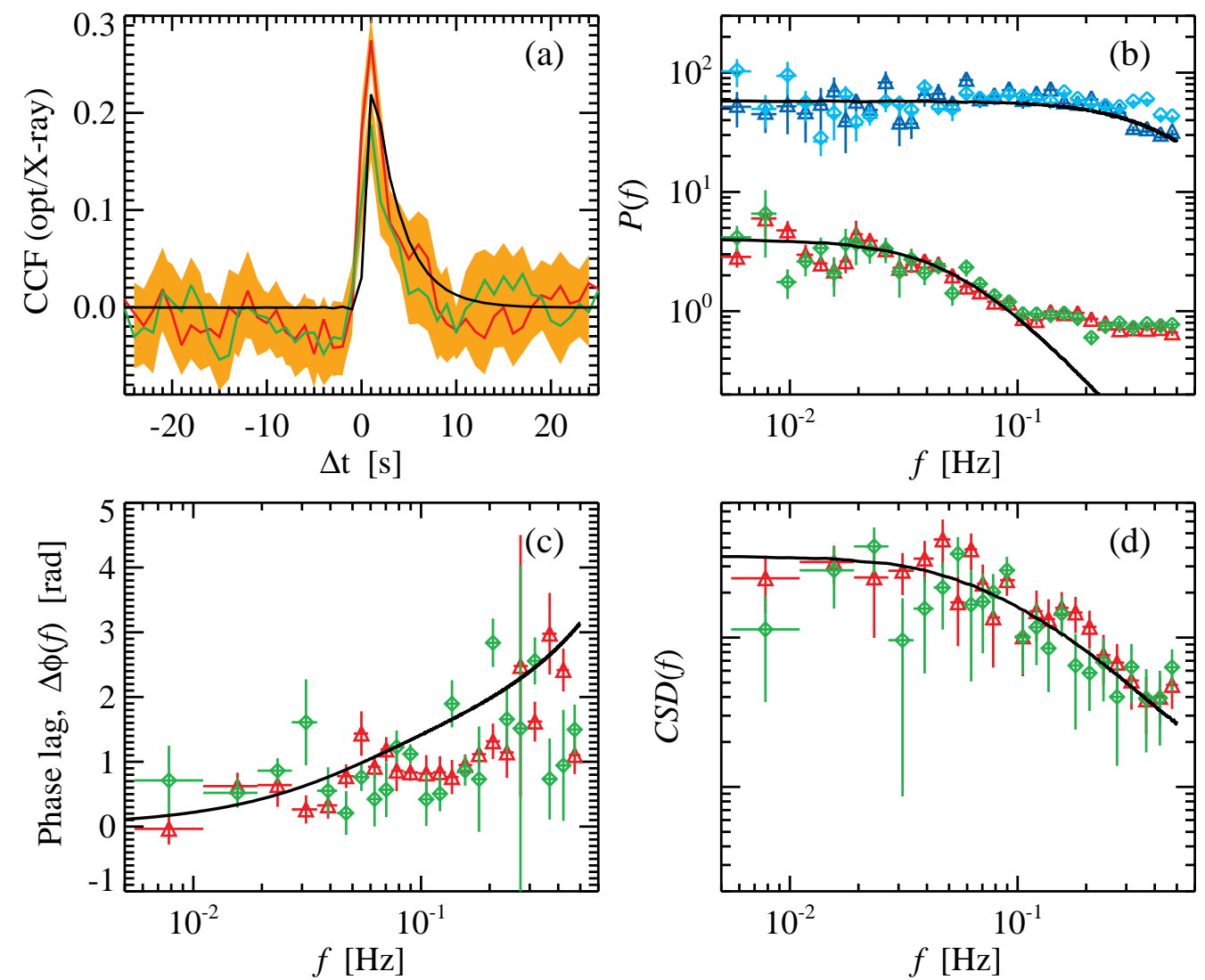

Figure 4. Characteristics of the light-curves observed during the outburst peak. (a) Optical/X-ray CCF from 2005 July 6 (red) and July 7 (green). The yellow stripe represents the errors. The peak on July 7 is reduced compared to that on July 6 , however the difference is marginally significant. (b) X-ray (top) and optical (bottom) PSDs from 2005 July 6 (blue and red triangles) and July 7 (cyan and green diamonds). (c) phase lag spectra and (d) cross-spectra from 2005 July 6 (red triangles) and July 7 (green diamonds). The model is shown with a solid black line.

mean square variability amplitude of the disc to that of the synchrotron component.

The irradiated disc light-curve $d(t)$ is delayed and smeared with respect to the X-ray light-curve $x(t)$, which we simulate by a convolution of the X-ray light-curve with the disc response function, which we approximate by a simple exponential (Poutanen 2002; Veledina et al. 2011b)

$r(t)=\left\{\begin{array}{cc}\exp \left[-\left(t-t_{1}\right) / t_{2}\right] / t_{2}, & t \geqslant t_{1}, \\ 0, & t<t_{1},\end{array}\right.$

its shape is controlled by two parameters: the delay $t_{1}$ and the decay time $t_{2}$. We note that the realistic transfer function should include a rather stable contribution of the disc and a variable contribution of the companion star, changing over an orbital period (see, e.g. fig. 2 of O'Brien et al. 2002). We consider this generic response function as a rough approximation having the least possible number of parameters.

The synchrotron radiation fluctuates in response to changing mass accretion rates, however, in the opposite sense to the X-rays: when the mass accretion rate increases, the $\mathrm{X}$-rays increase, but the synchrotron emission is suppressed because of the increased self-absorption. The optical emission is mostly produced at radii of about $15 R_{\mathrm{S}}$ or further (Veledina et al. 2013a), where the flow becomes effectively transparent to synchrotron. These radii are some- what larger than the characteristic region of major energy release, thus the synchrotron light-curve is expected to be smeared compared to the X-rays coming from synchrotron Comptonization, which we again simulate with the low-pass filter given by equation (1). On the other hand, the region where optical photons are generated is likely to be situated close to the disc truncation radius, so the damping frequency for disc Comptonization is expected to be similar to that of synchrotron emission. We fix the damping frequency for synchrotron and disc Comptonization to be the same. The characteristic damping frequency might differ substantially when the parameters of the hot flow change. The synchrotron emission is modulated at the QPO frequency as the hot flow precesses (Veledina et al. 2013b). Similarly to the $\mathrm{X}$-rays, the aperiodic synchrotron variability is multiplied by the QPO light-curve.

The quasi-periodic X-ray light-curve also modulates reprocessed emission at corresponding frequencies. However, the characteristic delay and profile shape depend on many unknown disc parameters (Veledina \& Poutanen 2015), and in order to obtain the optical and X-ray QPOs in phase (as observed in SWIFT J1753.5-0127, Veledina et al. 2015), fine-tuning of parameters is needed. We do not consider this possibility further.

The mathematical description of the picture introduced 

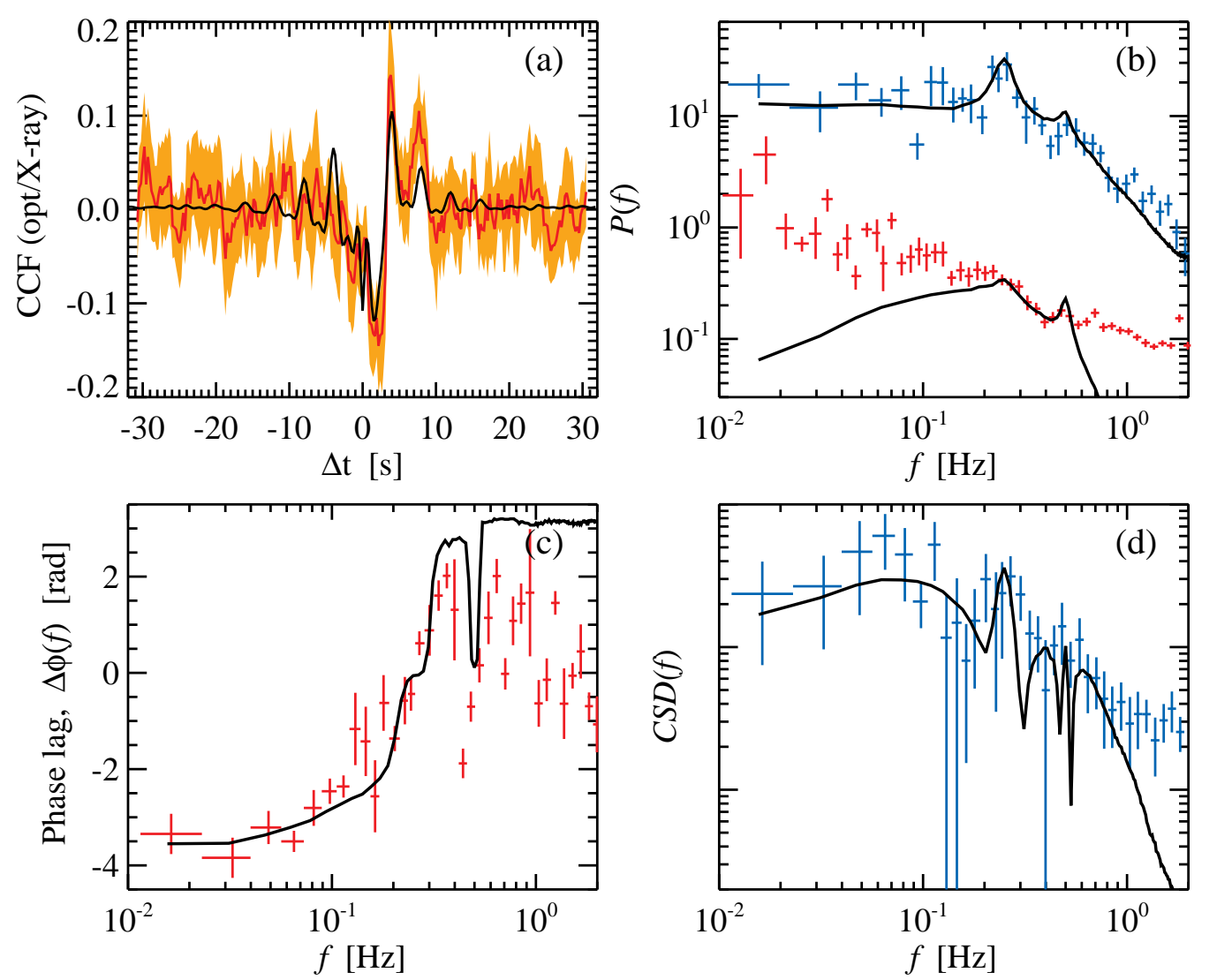

Figure 5. Characteristics of the light-curves observed during the decline stage of the outburst. (a) Optical/X-ray CCF (red) and its errors (yellow), (b) X-ray (blue, top) and optical (red, bottom) PSDs, (c) phase lag spectrum and (d) cross-spectral density. We subtract $2 \pi$ from the phase lags at frequencies below $\sim 0.07 \mathrm{~Hz}$ for clarity. The model is overplotted with a solid black line.

is

$s(t)=[1-\dot{m}(t) * h(t)]\left[1+\varepsilon_{\mathrm{o}} q(t)\right]-1$,

$d(t)=\left[\varepsilon_{\mathrm{m}} \dot{m}\left(t+t_{0}\right) * h(t)+\dot{m}(t)\right] * r(t)$,

where $\varepsilon_{\mathrm{o}}$ is a positive parameter responsible for the prominence of the QPO. The first equation describes the synchrotron light-curve and is similar to the hot-flow X-ray light-curve (the term in brackets in equation 2), apart from the sign in front of mass accretion rate light-curve $\dot{m}(t)$ and the presence of filter function $h(t)$. This minus sign denotes the anti-correlation of the synchrotron emission and the local mass accretion rate fluctuations, which leads to an anticorrelation of the hot flow synchrotron and X-ray variability. The plus signs in front of $\varepsilon_{\mathrm{x}}$ (equation 2) and $\varepsilon_{\mathrm{o}}$ (equation 4 ) reflect that the two QPOs come in phase. The term in brackets in the second equation is just the X-ray light-curve without the QPO.

\subsection{Modelling procedure and study of parameter space}

The light-curve of the mass accretion rate as measured in the region of X-ray production, $\dot{m}(t)$, is calculated from the prescribed PSD using Timmer \& Koenig (1995) algorithm, with zero mean. The PSD is described by a sum of Lorentzians of the form

$L_{i}(f)=\frac{r_{i}^{2} \Delta f_{i}}{\pi\left[\Delta f_{i}^{2}+\left(f-f_{i}\right)^{2}\right]}, \quad i=0,1,2$,

where $r_{i}$ describe their relative normalisations. The broadband variability is represented by one or two zero-centred Lorentzians $(i=1,2)$, thus $f_{1}=f_{2}=0$ (after Nowak et al. 1999). The QPO and its second harmonic are described by Lorentzians $(i=0)$ with central frequencies $f_{0}=f_{\mathrm{QPO}}$ and $f_{0}=2 f_{\mathrm{QPO}}$, respectively. We put the parameter $r_{0}=1$, so $\varepsilon_{\mathrm{x}}$ and $\varepsilon_{\mathrm{o}}$ determine the peak value of the Lorentzian. Several methods to simulate the light-curve with the aperiodic noise and the QPO were proposed (Burderi et al. 1997; Lazzati \& Stella 1997; Menna et al. 2003; Ingram \& Done 2012). We follow the latter approach and simulate the hot flow QPO from its PSD using the Timmer \& Koenig (1995) algorithm, with zero mean.

The disc to synchrotron ratio $r_{\mathrm{ds}}$ regulates the relative importance of the dip and the peak in the CCF. This parameter also affects the phase lags: for large $r_{\mathrm{ds}}$ the phase lags resemble those of the disc $(\Delta \phi$ increase with Fourier frequency), for smaller $r_{\mathrm{ds}}$ the phase lags resemble those of the synchrotron term $(\Delta \phi \sim \pi)$. The disc parameters $t_{1}$ and $t_{2}$ define the characteristic frequency of suppression of high frequencies in the disc PSD and the frequency above which the phase lags arising from reprocessing start to substantially deviate from zero. In the CCF, $t_{1}$ is responsible for the shift of the positive peak and $t_{2}$ determines its width. Parameter 

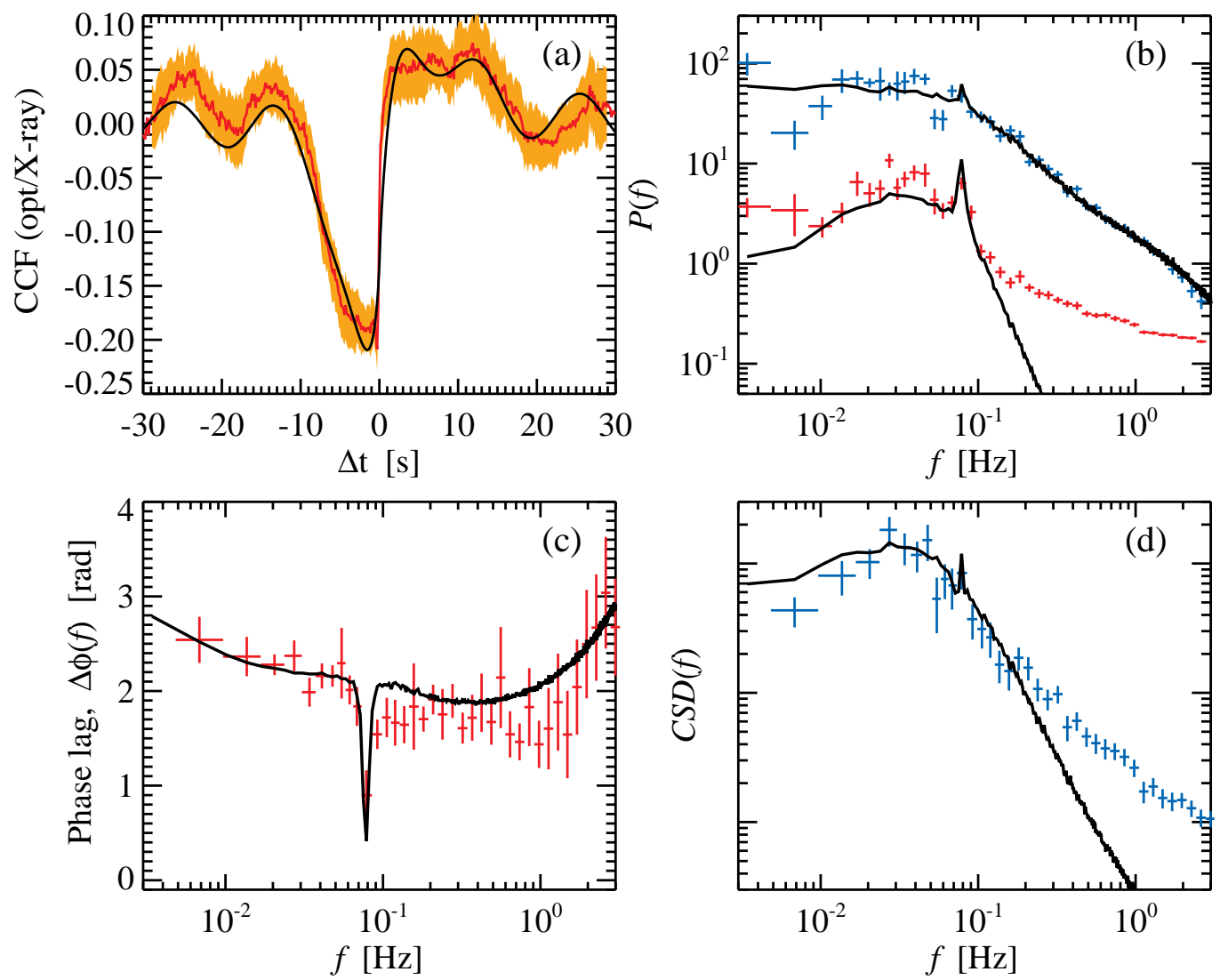

Figure 6. Characteristics of the light-curves observed during the outburst tail in 2007. (a) Optical/X-ray CCF (red) and its errors (yellow), (b) X-ray (blue, top) and optical (red, bottom) PSDs, (c) phase lag spectrum and (d) cross-spectral density. The model is overplotted with black solid line.

$f_{\text {filt }}$ acts on the dip width in the CCF: a smaller $f_{\text {filt }}$ gives a wider dip. The prominence of bumps in the X-ray PSD is regulated by the parameter $\varepsilon_{\mathrm{m}}$, which also affects the relative amplitude of dips in the CCF. Finally, $t_{0}$ determines the position of bumps in the X-ray PSD and the shift of the dip in the CCF (with respect to zero).

The parameters $\varepsilon_{\mathrm{x}}$ and $\varepsilon_{\mathrm{o}}$ regulate the QPO prominence in the PSDs, the wave amplitude in the CCF and the dip depth, as well as affecting the phase lags at $f_{\mathrm{QPO}}$. The QPO Lorentzian width $\Delta f_{\mathrm{QPO}}$ determines the characteristic timescale of coherent oscillations.

\section{RESULTS OF MODELLING}

The resulting CCF, X-ray and optical PSDs, phase lags and cross spectra are shown in Figs $4-7$ and the model parameters for equations (2) and (4) are listed in Table 1. The first six parameters $\left(r_{\mathrm{ds}}, t_{1}, t_{2}, f_{\text {filt }}, t_{0}\right.$ and $\left.\varepsilon_{\mathrm{m}}\right)$ describe the broadband variability. The following four parameters $\left(\varepsilon_{\mathrm{x}}\right.$, $\left.\varepsilon_{\mathrm{x}}, \Delta f_{0}, r_{0}\right)$ describe the appearance of the QPO, manifesting itself as waves in the CCF, narrow spikes in the PSDs, and the phase lags at the QPO's fundamental and harmonic frequencies.

At the peak of the outburst we see no QPOs and therefore we set $\varepsilon_{\mathrm{x}}=0$. Furthermore, there is no indication for a precognition dip. In the context of the model this suggests a minor contribution of synchrotron emission and thus $r_{\mathrm{ds}} \gg 1$. The X-rays are dominated by the disc Comptonization, hence we take $\varepsilon_{\mathrm{m}} \gg 1$ and $t_{0}=0$. Finally the filter function can be omitted. Under these conditions, the equations for the X-ray and optical light-curves reduce to $x(t)=\dot{m}(t), o(t)=\dot{m}(t) * r(t)$. We describe both the July 6 and 7 data with one set of model parameters. The model for outburst peak is shown in Fig. 4.

The CCFs are well reproduced in the simple model of disc reprocessing. The same conclusion was also reached by Hynes et al. (2009), where more realistic disc transfer functions (O'Brien et al. 2002) were used to model the CCF. The phase lag spectrum demonstrates a slowly increasing function of frequency and is in agreement with model predictions. The optical PSD is reproduced up to $f \sim 0.1 \mathrm{~Hz}$, above which the model significantly underestimates the data. We attribute this inconsistency to the presence of highfrequency atmospheric noise. We note that the cross spectrum is well reproduced up to Nyquist frequency, suggesting that the model reproduces the entire correlated signal.

The outburst decline stage is described by the equations (2) and (4) with all parameters being non-zero. There are simultaneously two components in both the optical and the X-ray wavelengths. The $\mathrm{CCF}$ demonstrates strong coupling of the broadband spectral variability to the QPO (Fig. 5), which now has two harmonics. Both positive peaks are produced by the QPO. There are two dips in the CCF: one at time lag $\sim-1 \mathrm{~s}$ and another at $\sim 2 \mathrm{~s}$. They are 

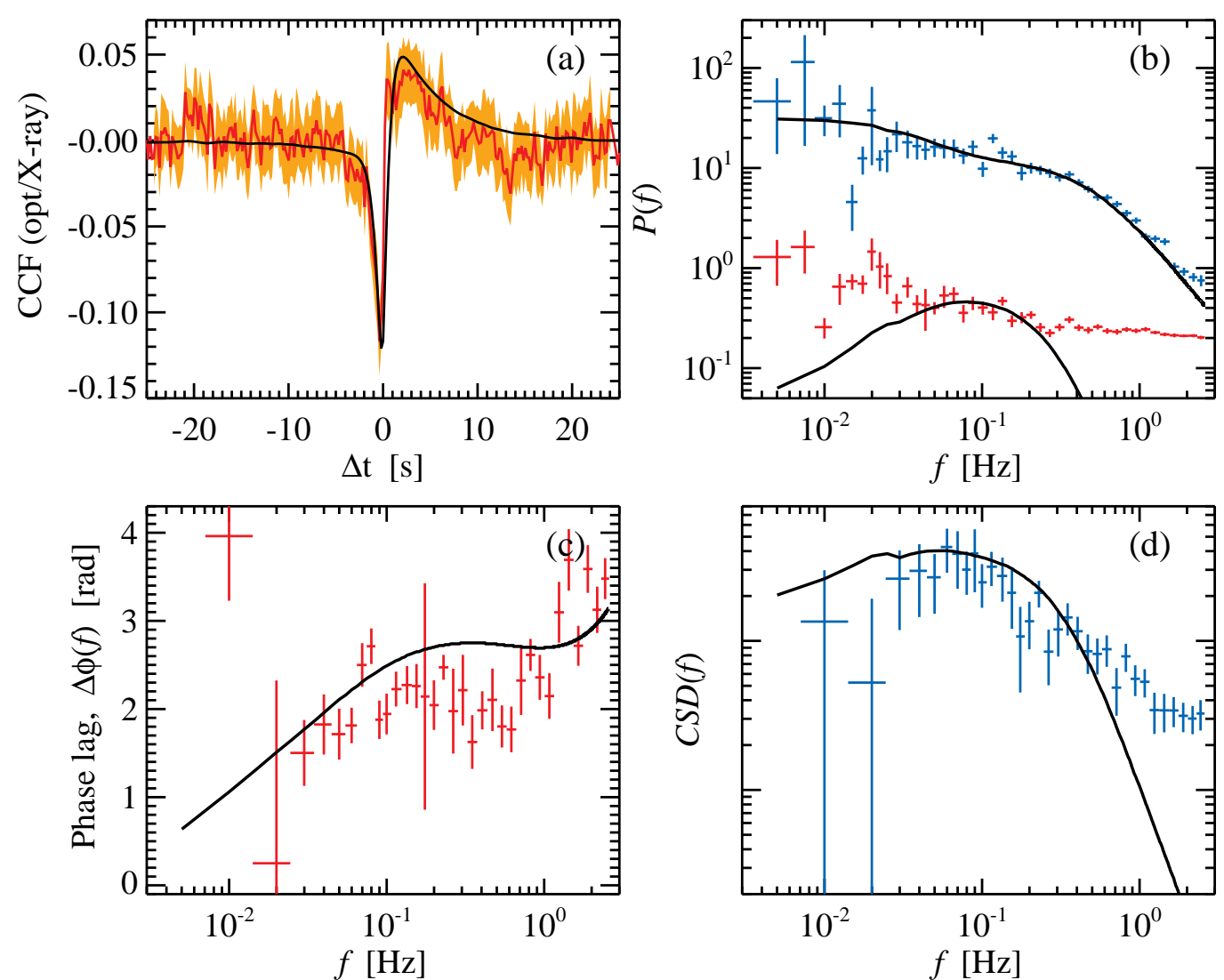

Figure 7. Characteristics of the light-curves observed during the outburst tail in 2008. (a) Optical/X-ray CCF (red) and its errors (yellow), (b) X-ray (blue, top) and optical (red, bottom) PSDs, (c) optical/X-ray phase lag spectrum and (d) cross-spectral density. The model is shown with a solid black line.

Table 1. Parameters of numerical modelling.

\begin{tabular}{|c|c|c|c|c|}
\hline Parameter & 2005 July 6,7 & 2005 Aug & 2007 & 2008 \\
\hline$r_{\mathrm{ds}}$ & $\infty$ & 0.4 & 0.8 & 0.8 \\
\hline$t_{1}(\mathrm{~s})$ & 0.15 & 0.15 & 0.15 & 0.15 \\
\hline$t_{2}(\mathrm{~s})$ & 3.0 & 3.0 & 4.5 & 3.0 \\
\hline$f_{\text {filt }}(\mathrm{Hz})$ & - & 0.45 & 0.05 & 0.3 \\
\hline$t_{0}(\mathrm{~s})$ & - & 1.8 & - & - \\
\hline$\varepsilon_{\mathrm{m}}$ & - & 1.8 & - & - \\
\hline$\varepsilon_{\mathrm{x}}$ & - & $1.8,0.5$ & 0.4 & - \\
\hline$\varepsilon_{\mathrm{o}}$ & - & $0.1,0.13$ & 0.4 & - \\
\hline$f_{0}(\mathrm{~Hz})$ & - & 0.25 & 0.078 & - \\
\hline$\Delta f_{0}(\mathrm{~Hz})$ & - & 0.03 & 0.003 & - \\
\hline \multicolumn{5}{|c|}{ X-ray broadband noise parameters } \\
\hline$\Delta f_{1}(\mathrm{~Hz})$ & 0.46 & 0.3 & 0.095 & 0.03 \\
\hline$r_{1}$ & 1.0 & 1.0 & 1.3 & 0.5 \\
\hline$\Delta f_{2}(\mathrm{~Hz})$ & - & - & 1.5 & 0.5 \\
\hline$r_{2}$ & - & - & 0.9 & 0.15 \\
\hline
\end{tabular}

produced by the anti-correlation of the synchrotron optical emission with the two X-ray components. Although we did not introduce any time-delays between the synchrotron and its Comptonization, the dip appears at somewhat negative lags due to a significant contribution of the (positively correlated) irradiated disc emission at $\Delta t \sim 0$, partially can- celling the negative contribution of the synchrotron emission at zero lag.

The X-ray model PSD demonstrates a peak at $0.6 \mathrm{~Hz}$ arising from the interference of the two X-ray terms (Veledina 2016). The optical model PSD is again somewhat different from the observed one at high frequencies, but the cross spectrum is well reproduced up to $\sim 1 \mathrm{~Hz}$. The rapid increase of phase lags with frequency appears in our model (mainly) because of the delay between the optical synchrotron lightcurve with respect to the X-ray light-curve arising from disc Comptonization. The increase of phase lags becomes somewhat more rapid when we include the QPO. A sharp drop of phase lags seen at the double QPO frequency is due to the presence of the second QPO harmonic, which may arise from the anisotropy of the synchrotron radiation.

The 2007 dataset shows no indication of multiple dips and peaks in the CCF. We interpret this as an indication of one component contributing to X-rays, namely, the synchrotron Comptonization, and put $\varepsilon_{\mathrm{m}}=0$. The model has the first QPO harmonic revealing itself in both the CCF and in the phase lags (Fig. 6). We see that the phase lags are almost independent of frequency, as explained by the joint contribution of the synchrotron (having $\Delta \phi \sim \pi$ ) and the disc terms ( $\Delta \phi \sim 0$ in the frequency range of interest). At the QPO frequency the phase lags demonstrate a sharp dip, as the X-ray and optical QPOs come into phase. The cross-spectrum is reproduced up to $\sim 0.2 \mathrm{~Hz}$.

The 2008 dataset is described by the model with $\varepsilon_{\mathrm{m}}=0$ 


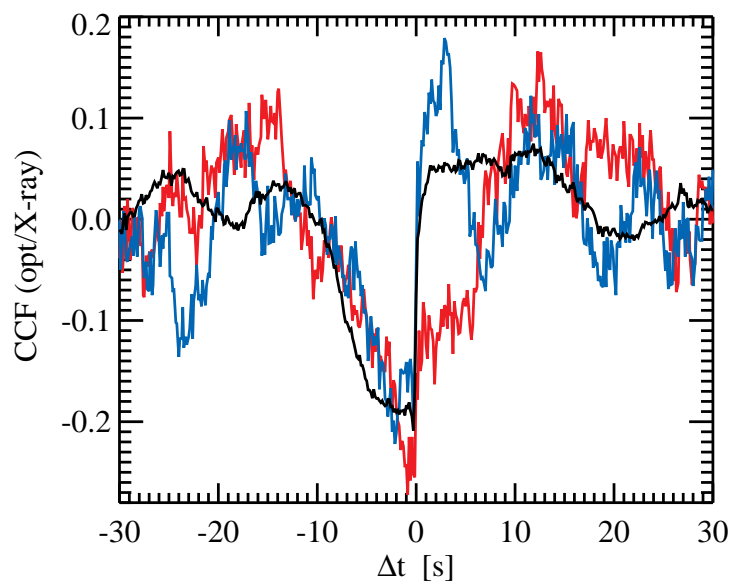

Figure 8. CCFs from two segments of 2007 data (red, blue) and the average CCF (black). Variations of the shape are apparent, in particular, at small positive lags.

(only synchrotron Comptonization contributing to X-rays), and without QPOs, $\varepsilon_{\mathrm{x}}=0, \varepsilon_{\mathrm{o}}=0$, see Fig. 7. The phase lags demonstrate a kink at $\sim 0.4 \mathrm{~Hz}$. Noting that our model has a characteristic frequency of synchrotron emission suppression $f_{\text {filt }}=0.3 \mathrm{~Hz}$, we interpret this kink as a transition from a phase lag spectrum dominated by synchrotron (for $f \lesssim$ $0.3 \mathrm{~Hz})$ to one dominated by the disc $(f \gtrsim 0.4 \mathrm{~Hz})$. We again see that the optical PSD and CCF demonstrate strong noise, hence we use the cross-spectral density spectrum to check that the correlated (intrinsic) signal is well reproduced. The cross-spectrum is reproduced up to $f \sim 0.7 \mathrm{~Hz}$.

\section{DISCUSSION}

\subsection{Deviations of the model from the data}

We find deviations of the model from the data in the optical PSDs at high frequencies (above about $0.5 \mathrm{~Hz}$ ) in all datasets. We suggest these inconsistencies are mostly due to atmospheric noise. Scintillation due to refraction from changing turbulent cells in the atmosphere contributes to higher Fourier frequencies, complementing the contribution of Poisson noise here. Differential extinction variations may contribute to noise at lower frequencies (these effects are likely seen at low frequencies of the optical PSD from the outburst decline).

Considering the good agreement between the observed and modelled CCFs and phase-lags, the deviations of the cross-spectra are more puzzling. We find that the excess power at high frequencies can be accounted for by a more sophisticated shape of the filter function for synchrotron radiation (which determines the PSD shape at high frequencies). For example, by adding a constant to the filter (equation 1) we can achieve good agreement between the modelled and observed cross spectra. This additional constant accounts for the fact that the synchrotron radiation is likely distributed within the flow, partially coming from its outer parts (and thus smeared) and partially originating from the regions where the bulk of the X-rays are emitted. The CCF is also somewhat better described in this case, as now we are able to account for the small negative spike seen at time lags close to zero in Fig. 6a. However, the model phase lags

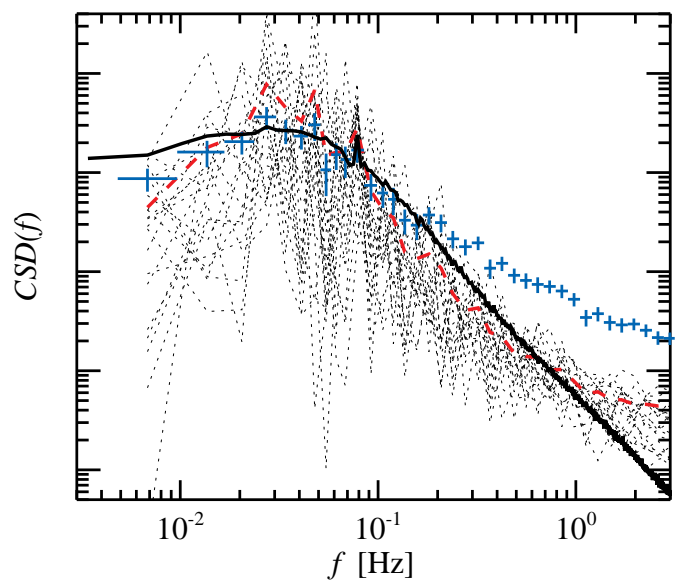

Figure 9. Cross-spectra from the 2007 data, calculated by the vector averaging of CSDs from different segments (blue crosses, same as in Fig. 6, but scaled up by a factor of 20) and by averaging of CSD amplitudes of different segments (red dashed line). The latter describes a typical CSD from one segment. Cross-spectra from each segment are shown with black dotted lines. The model (black solid line) describes the typical CSD better than the one obtained from vector averaging.

then deviate somewhat more from the data, suggesting we need a more realistic shape for the response function.

Another reason for the discrepancy might be that we have implicitly assumed that the parameters do not change within one epoch. However, the observed CCF shows large shape variations even during one observational run (see Fig. 8). Each segment CCF can be reproduced by the model, but requires different parameters. Primarily, we expect the disc to synchrotron ratio $r_{\mathrm{ds}}$ to change. We note that the (model) CCFs obtained from averaging of CCFs computed with different parameters is not equivalent to the CCF calculated from one parameter set, even if the parameters are the average ones.

To investigate the reason for the appearance of the highfrequency power in cross-spectra we checked their shape in every segment. In Fig. 9 we show the cross-spectrum obtained from vector averaging, as before (blue crosses), and each segment CSD (black dotted lines). We see that their shapes differ substantially at high frequencies. This discrepancy is caused by the reduction of coherence at low frequencies, compared to higher ones and is seen as larger spread (at lower frequencies) between values from different segments (the two to three orders of magnitude spread at lower frequencies is opposed to an order of magnitude spread at higher frequencies). Hence, the vector averaging effectively reduces the power at lower frequencies more, as compared to higher frequencies.

From the model point of view, the large spread at lower frequencies is likely to be the result of varying disc to synchrotron ratio within one observation. Because the interplay of the two components is most prominent at lower frequencies, the spreading is higher here. In fact, the change of the CCF shape (Fig. 8) is a manifestation of the same phenomenon as the reduction of cross-spectral power at low frequencies. We note that the model cross-spectrum (black solid line in Fig. 9) reproduces the shape of the crossspectrum of a typical segment (red dashed line) substantially better than the one obtained from vector averaging. 

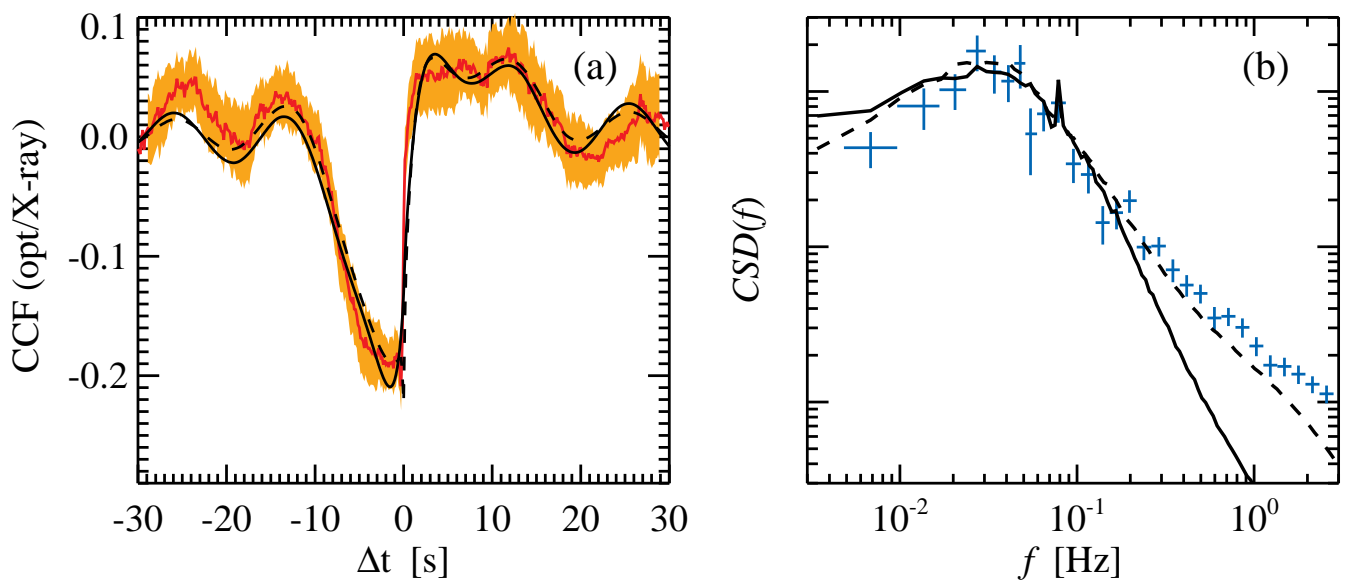

Figure 10. CCF (a) and CSDs (b) of the 2007 dataset, as in Fig. 6. Black solid lines correspond to the model with constant parameters, dashed lines correspond to the model with varying disc to synchrotron ratio ( $r_{\mathrm{ds}}$ parameter), all other parameters are fixed and same as for the solid line.

To further investigate this hypothesis we perform modelling allowing the disc to synchrotron ratio change. We assume that the $r_{\mathrm{ds}}$ takes values between 0 and 1.6 with flat probability distribution, while other parameters are fixed to values from Table 1 . For each value, we simulate the optical light-curve, compute CCF and CDS and then average these values over 1500 different realisations. The resulting characteristics are compared to those modelled for constant parameters and with the data in Fig. 10. We find that the shape of the cross spectrum has dramatically changed, now exhibiting the missing power at higher frequencies. Moreover, the CCF now exhibits a small spike at negative lags, similar to that observed.

We note that the standard procedure of improving signal-to-noise by averaging over a number of data segments (van der Klis 1989) assumes that all segments have the same statistical properties, i.e. the time series is stationary. We see that this assumption is violated in the cases we are considering (Fig. 8), warning similar problems for optical data in other systems. Further data processing should take this limitation into account.

\subsection{Evolution of parameters}

Some restrictions on the parameters can be imposed from physical arguments. The reprocessed disc emission is mostly coming from the outer, flared rim of the disc, thus we expect $t_{1} \gtrsim R_{\mathrm{d}}(1-\sin i) / c$ (where $R_{\mathrm{d}}$ is the disc outer radius, Poutanen 2002). For $i \lesssim 75^{\circ}$ (the maximal inclination observed for a BH binary, Casares \& Jonker 2014) we get $t_{1} \gtrsim 0.07 \mathrm{~s}$. The decay time $t_{2}$ is connected to the characteristic size of the disc. Taking the semi-major axis $a=10^{11} \mathrm{~cm}$ (Neustroev et al. 2014), we expect a maximal outer disc radius $R_{\mathrm{d}}=6 \times 10^{10} \mathrm{~cm}$ (equal to the tidal truncation radius), constraining $t_{2} \sim 2 \mathrm{~s}$. The decay time obtained for the 2007 dataset is somewhat larger. The reason for that might be the increase of the delays from the companion star due to a change of the orbital phase (see fig. 2 of O'Brien et al. 2002), which is completely ignored in our model.

To explain the evolution of the temporal properties, we utilise the truncated disc scenario and consider the appearance of an additional optical (synchrotron) component towards the tail of the outburst. In our model, the truncation radius is connected to the synchrotron filtering frequency, which is found to have a minimal value in 2007 . If they are not a result of noise contamination, our findings suggest that the truncation radius is small at the outburst peak, perhaps, smaller than $10 R_{\mathrm{S}}$ (when we see no synchrotron emission), increases until 2007, when we see it at maximum, and decreases again in 2008. The evolution of timing properties is consistent, in this scenario, with the spectral evolution, see Fig. 11. The total X-ray flux drops and the spectrum hardens from 2005 Jul 6 to 2007 June 13, but then a re-brightening and a somewhat softer spectrum appears on 2008 Aug 10. Moreover, a scenario of increasing truncation radius and development of the hot accretion flow in SWIFT J1753.5-0127 is supported by broadband (optical to X-ray) spectral modelling (fig. 8 of Kajava et al. 2016).

The evolution of the UV/optical fluxes during the first months of the outburst are also peculiar. While the Xray flux dropped by a factor of 15 , the fluxes in the UV range and in the B-filter drop by a factor of $\sim 2$. This behaviour was shown to be in conflict with reprocessing scenario (Chiang et al. 2010). This either suggests a dramatic change of reprocessing properties (such as change of albedo) or is a signature of the appearance of an additional component (likely synchrotron radiation locally produced in the hot flow). The latter explanation is more favoured by our findings.

We note that the disc to synchrotron ratio, $r_{\mathrm{ds}}$, has a low value during the decline stage, when the hot flow appears. The ratio then increases in the outburst tail, indicating the synchrotron flux is higher, relative to the disc, in the decline stage than in the tail. This trend is explained by the different scaling with the X-ray luminosity. For the long-term variations, such as the characteristic timescales of the outburst, the synchrotron scales roughly as $L_{\text {syn }} \propto L_{\mathrm{X}}$ (as the spectral slope of the synchrotron self-Compton spectrum remains constant for different luminosities; see fig. $7 \mathrm{~b}$ of Veledina, Vurm \& Poutanen 2011a). The observed disc 


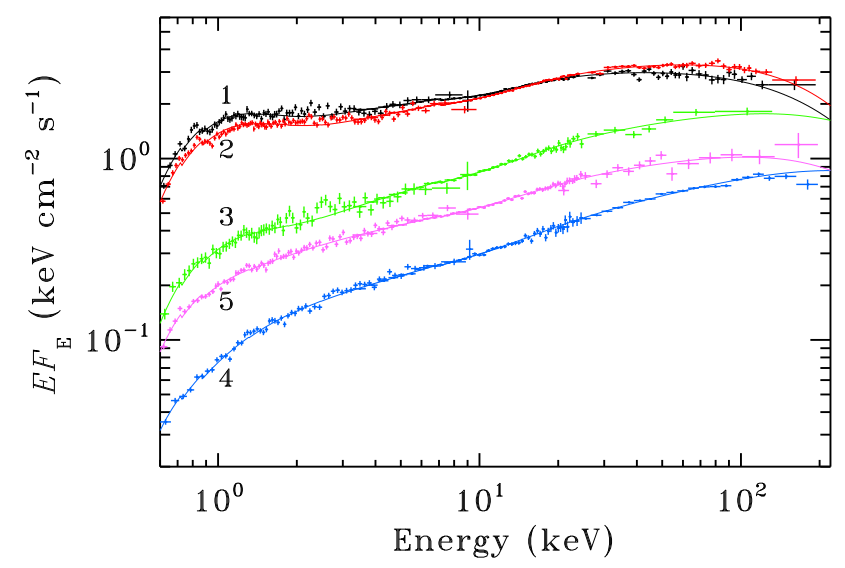

Figure 11. X-ray spectra of the considered epochs: 2005 July 6 (1, black), July 7 (2, red), Aug 9 (3, green), 2007 June 13 (4, blue), 2008 Aug 10 (5, magenta). The spectra 1 and 2 are fitted using TBABS $\times($ DISKBB + COMPPS) (Wilms, Allen \& McCray 2000; Mitsuda et al., 1984; Poutanen \& Svensson 1996, respectively). For spectra $3-5$, only TBABS $\times$ COMPPS was used. The data are from Swift/XRT, RXTE/PCA and RXTE/HEXTE (spectra 1, 2,3 ) or INTEGRAL/ISGRI (spectra 4 and 5).

spectrum likely falls in the transition between the RayleighJeans regime where $L_{\mathrm{d}} \propto T_{\mathrm{d}} \propto L_{\mathrm{X}}^{1 / 4}$ (where $T_{\mathrm{d}}$ is the irradiated disc temperature) and the blackbody peak with $L_{\mathrm{d}} \propto L_{\mathrm{X}}$, so we can roughly approximate $L_{\mathrm{d}} \propto L_{\mathrm{X}}^{1 / 2}$. The ratio $L_{\mathrm{d}} / L_{\mathrm{s}} \propto L_{\mathrm{X}}^{-1 / 2}$ increases as the X-ray luminosity decreases. A similar trend was observed in the $\mathrm{BH}$ binary XTE J1550-564, the spectral evolution of which allowed the contributions of these two components to be separated (Poutanen, Veledina \& Revnivtsev 2014). Hence, the results of our timing analysis are in agreement with expectations from spectral studies.

\section{CONCLUSIONS}

For the first time we model the complex evolution of the optical/X-ray timing properties of the 2005 outburst of the BH binary SWIFT J1753.5-0127. The shape of the optical/X-ray cross-correlation function demonstrates a single peak at the time of the outburst peak, which is then replaced by multiple dips and peaks during the outburst decline and a single dip plus peak structure in the outburst tail (see Fig. 2). We interpret this behaviour as the appearance of synchrotron emission from the hot flow towards the end of the outburst. This scenario is also supported by the previously reported evolution of the spectral properties, with disc Comptonization dominating the X-ray spectrum at the peak and synchrotron Comptonization dominating in the tail.

We propose a quantitative model describing the characteristics of each stage. For the first time, the model is capable of explaining the CCF through the decline. We show that coupling with the QPO significantly alters the shape of CCF. The tentative presence of the QPO harmonic can be seen in the X-rays, however, its significance is very low. We find that a second QPO harmonic is needed to account for the phase lags at double the QPO frequency. Similar to the first harmonic, X-ray and optical QPOs are intrinsically connected and the phase shift is zero for both harmonics.

To describe the complex phase lags in the decline stage, two components in X-rays are needed: one coming from the disc- and the other from synchrotron Comptonization. Interestingly, such a two-component X-ray light-curve naturally gives the humps in the X-ray power spectrum, which had to be modelled with additional Lorentzian components or QPOs in previous works.

We note that the optical/X-ray cross spectra show excess power at high frequencies. We attribute this feature to the limitations of the standard procedure of filtering out the noise by averaging over the data segments, which assumes that the statistical characteristics are time-invariant. We show that the trend of artificial power increase at high frequencies can be reproduced by simulating a number of CCFs with varying parameters.

We find that the CCF shape is an independent indicator of the accretion geometry. We show that the study of correlated optical/X-ray variability allows us to probe radiative mechanisms operating in the immediate vicinity of the black hole. In the future, systematic study of the changing CCF shape during the outbursts of X-ray binaries may finally shed light on the physical processes accompanying state transitions.

\section{ACKNOWLEDGEMENTS}

AV thanks A. Zdziarski for the comments on the manuscript. The work was supported by the Academy of Finland grant 268740 (AV, JP), the Foundations' Professor Pool and the Finnish Cultural Foundation (JP). JJEK acknowledges support from the ESA research fellowship programme. MGR and SST were supported by the Russian Science Foundation grant 14-12-01287.

\section{REFERENCES}

Arévalo P., Uttley P., 2006, MNRAS, 367, 801

Basak R., Zdziarski A. A., 2016, MNRAS, 458, 2199

Belloni T. M., Stella L., 2014, Space Sci. Rev., 183, 43

Belloni T., Psaltis D., van der Klis M., 2002, ApJ, 572, 392

Bendat J. S., Piersol A. G., 1986, Random Data: Analysis and Measurement Procedures. Wiley, New York

Burderi L., Robba N. R., La Barbera N., Guainazzi M., 1997, ApJ, 481, 943

Casares J., Jonker P. G., 2014, Space Sci. Rev., 183, 223

Casella P., et al., 2010, MNRAS, 404, L21

Chaty S., Haswell C. A., Malzac J., Hynes R. I., Shrader C. R., Cui W., 2003, MNRAS, 346, 689

Chiang C. Y., Done C., Still M., Godet O., 2010, MNRAS, 403, 1102

Churazov E., Gilfanov M., Revnivtsev M., 2001, MNRAS, 321,759

De Marco B., Ponti G., 2016, ApJ, 826, 70

De Marco B., Ponti G., Muñoz-Darias T., Nandra K., 2015, ApJ, 814,50

Dhillon V. S., et al., 2007, MNRAS, 378, 825

Done C., Gierliński M., Kubota A., 2007, A\&A Rev., 15, 1

Durant M., Gandhi P., Shahbaz T., Fabian A. P., Miller J., Dhillon V. S., Marsh T. R., 2008, ApJ, 682, L45

Durant M., Gandhi P., Shahbaz T., Peralta H. H., Dhillon V. S., 2009, MNRAS, 392, 309 
Durant M., et al., 2011, MNRAS, 410, 2329

Esin A. A., McClintock J. E., Narayan R., 1997, ApJ, 489, 865

Fragile P. C., Blaes O. M., Anninos P., Salmonson J. D., 2007, ApJ, 668, 417

Froning C. S., Maccarone T. J., France K., Winter L., Robinson E. L., Hynes R. I., Lewis F., 2014, ApJ, 780, 48

Frontera F., et al., 2001, ApJ, 561, 1006

Gandhi P., et al., 2008, MNRAS, 390, L29

Gandhi P., et al., 2010, MNRAS, 407, 2166

Gilfanov M., 2010, in T. Belloni ed., Lecture Notes in Physics Vol. 794, The Jet Paradigm. Springer Verlag, Berlin, pp 1751 (arXiv:0909.2567), doi:10.1007/978-3-540-76937-8_2

Gilfanov M., Churazov E., Revnivtsev M., 2000, MNRAS, 316,923

Hynes R. I., O'Brien K., Horne K., Chen W., Haswell C. A., 1998, MNRAS, 299, L37

Hynes R. I., et al., 2003, MNRAS, 345, 292

Hynes R. I., O'Brien K., Mullally F., Ashcraft T., 2009, MNRAS, 399,281

Ingram A., Done C., 2011, MNRAS, 415, 2323

Ingram A., Done C., 2012, MNRAS, 419, 2369

Ingram A., Done C., Fragile P. C., 2009, MNRAS, 397, L101

Ingram A., van der Klis M., Middleton M., Done C., Altamirano D., Heil L., Uttley P., Axelsson M., 2016, MNRAS, 461, 1967

Kajava J. J. E., Veledina A., Tsygankov S., Neustroev V., 2016, A\&A, 591, A66

Kalamkar M., Casella P., Uttley P., O’Brien K., Russell D., Maccarone T., van der Klis M., Vincentelli F., 2016, MNRAS,

Kanbach G., Straubmeier C., Spruit H. C., Belloni T., 2001, Nature, 414, 180

Kotov O., Churazov E., Gilfanov M., 2001, MNRAS, 327, 799

Lazzati D., Stella L., 1997, ApJ, 476, 267

Leahy D. A., Darbro W., Elsner R. F., Weisskopf M. C., Kahn S., Sutherland P. G., Grindlay J. E., 1983, ApJ, 266, 160

Lyubarskii Y. E., 1997, MNRAS, 292, 679

Malzac J., Belmont R., 2009, MNRAS, 392, 570

Menna M. T., Burderi L., Stella L., Robba N., van der Klis M., 2003, ApJ, 589, 503

Mitsuda K., et al., 1984, PASJ, 36, 741

Motch C., Ricketts M. J., Page C. G., Ilovaisky S. A., Chevalier C., 1983, A\&A, 119, 171

Nather R. E., Mukadam A. S., 2004, ApJ, 605, 846

Neustroev V. V., Veledina A., Poutanen J., Zharikov S. V., Tsygankov S. S., Sjoberg G., Kajava J. J. E., 2014, MNRAS, 445,2424

Nowak M. A., Wilms J., Dove J. B., 1999, ApJ, 517, 355

O'Brien K., 2008, in Phelan D., Ryan O., Shearer A., eds, Astrophysics and Space Science Library Vol. 351, Astrophysics and Space Science Library. p. 187 (arXiv:0708.1629), doi:10.1007/978-1-4020-6518-7_10

O’Brien K., Horne K., Hynes R. I., Chen W., Haswell C. A., Still M. D., 2002, MNRAS, 334, 426

Plant D. S., Fender R. P., Ponti G., Muñoz-Darias T., Coriat M., 2015, A\&A, 573, A120

Poutanen J., 2002, MNRAS, 332, 257

Poutanen J., Svensson R., 1996, ApJ, 470, 249

Poutanen J., Veledina A., 2014, Space Sci. Rev., 183, 61

Poutanen J., Vurm I., 2009, ApJ, 690, L97

Poutanen J., Krolik J. H., Ryde F., 1997, MNRAS, 292, L21

Poutanen J., Veledina A., Revnivtsev M. G., 2014, MNRAS, 445,3987

Rahoui F., et al., 2015, ApJ, 810, 161

Remillard R. A., McClintock J. E., 2006, ARA\&A, 44, 49

Revnivtsev M., Gilfanov M., Churazov E., 1999, A\&A, 347, L23

Revnivtsev M., Gilfanov M., Churazov E., 2001, A\&A, 380, 520

Shaw A. W., et al., 2016, MNRAS, 458, 1636

Shaw A. W., et al., 2016b, The Astronomer's Telegram, 8782
Timmer J., Koenig M., 1995, A\&A, 300, 707

Tomsick J. A., et al., 2015, ApJ, 808, 85

Veledina A., 2016, preprint, (arXiv:1610.00431)

Veledina A., Poutanen J., 2015, MNRAS, 448, 939

Veledina A., Vurm I., Poutanen J., 2011a, MNRAS, 414, 3330

Veledina A., Poutanen J., Vurm I., 2011b, ApJ, 737, L17

Veledina A., Poutanen J., Vurm I., 2013a, MNRAS, 430, 3196

Veledina A., Poutanen J., Ingram A., 2013b, ApJ, 778, 165

Veledina A., Revnivtsev M. G., Durant M., Gandhi P., Poutanen J., 2015, MNRAS, 454, 2855

Wilms J., Allen A., McCray R., 2000, ApJ, 542, 914

Yoshikawa A., Yamada S., Nakahira S., Matsuoka M., Negoro H., Mihara T., Tamagawa T., 2015, PASJ, 67, 11

Zdziarski A. A., Gierliński M., 2004, Progr. Theor. Phys. Suppl., 155,99

van der Klis M., 1989, in Ögelman H., van den Heuvel E. P. J., eds, NATO Advanced Science Institutes (ASI) Series C Vol. 262, NATO Advanced Science Institutes (ASI) Series C. p. 27 